\title{
How Can we Treat Recurrent Herpes Virus Infection Without the Use of Antiviral Drugs?
}

\section{Huang WL}

Infectious disease doctor, general practitioner, nutrition doctor, medical acupuncturist, pain management doctor, Medical Acupuncture and Pain Management Clinic, Franca, São Paulo, Brazil

*Corresponding Author: Huang Wei Ling, Infectious disease doctor, general practitioner, nutrition doctor, medical acupuncturist, pain management doctor, Medical Acupuncture and Pain Management Clinic, Franca, São Paulo, Brazil.

Received: June 14, 2019; Published: July 26, 2019

DOI: $10.31080 /$ ASMS.2019.03.0363

\begin{abstract}
Statement of the problem: The herpes virus manifests as an eruption of red, painful blisters or sores in the skin, genitals, lips or eyes. In Western medicine, the causative symptoms are related to herpes simplex 1, herpes simplex 2, and varicella-zoster viruses, often combined with a weakening of immune function. In Traditional Chinese Medicine (TCM), this entire process is indicative of Fire Toxin and Damp Heat.

Purpose: To demonstrate that recurrent herpes virus infections can be treated without the use of antiviral drugs.

Methods: Two case reports. The first, from an 8-year-old girl with recurrent periocular lesions on the right eye, with edema formation, small blisters, hyperemia and hyperchromic spots. The symptoms were treated several times with topical and systemic antiviral medications, with the condition showing improvement only shortly. The second case report, from a 68-year-old woman, with a condition of recurrent genital herpes virus type 2 , which would not improve with the use of antiviral medication. Both patients were referred to an infectologist with a TCM background, who restructured the patient's dietary habits, recommending that all hot energy foods should be avoided. Auricular acupuncture sessions with apex ear bloodletting were also performed.

Results: A complete improvement of the conditions of both patients was obtained without using any antiviral drugs.

Conclusion: It is possible to treat recurrent herpes virus infection without the use of antiviral drugs. To this end, it is important to see the patient as a whole and treat their energy imbalances by withdrawing their Heat process through acupuncture and dietary counselling.
\end{abstract}

Keywords: Herpes Virus; Antiviral Drugs; Chinese Dietary Therapy; Acupuncture; Traditional Chinese Medicine

\section{Introduction}

Infectious associated with the Herpes simplex virus are among the most frequent infections found in humans [1].

There are two types of Herpes simplex virus infections. The type 1 of the virus is usually associated with orolabial infections, while type 2 is associated with genital and newborn infections. [1]. Studies point out that almost $90 \%$ of the world's population is exposed to Type 1 herpes, usually during childhood.
Despite being usually associated with orolabial infections, type 1 genital infections are becoming more common in the last decades. A study from Nelson LS Lee, entitled Herpes Simplex and Zoster, pointed out that in recent report, the proportion type 1 Herpes appearing as anogenital increased from $<10 \%$ in the 1980 's to $35 \%$ in the 1990's to 2000's [2].

The Varicella Zoster Virus is also a herpes virus. It is a painful red rash with blisters. Being the same virus that causes the chicken pox, the presentation of the virus is related to the contraction of the 
chicken pox. The virus can only be passed to others who have not had chicken pox. Someone newly infected with the herpes zoster virus will then develop chicken pox.

Also, according to the study of Nelson LS Lee, the indication of Herpes infections is the development of several "painful vesicles on an erythematous base involving a mucocutaneous site. The vesicles ulcerate and then heal by re-epithelialization.".

Both virus type 1 and 2 can appear as genital herpes, HSV proctitis and HSV eye infections. The disease may also become recurrent and the virus can present resistance to the treatment [2].

The Type 1 Herpes simplex virus is usually spread through direct contact with contaminated material such as saliva or other body secretions. [3] Type 2 Herpes virus simplex infection is spread through sexual contact. Even when asymptomatic, the disease can still be transmitted [4].

A study of the USA demonstrated that the incidence of genital herpes has been increasing among young adults in the country, probably because the disease is mainly associated with oral sex, more commonly practiced by young adults [4].

When Type 1 Herpes virus simplex affect the eye, the patient is at risk for more severe problems. These issues may include glaucoma, cataracts, double vision and scarring of the eyelid or cornea. That is why it was so important to treat the first patient as fast as possible, so the virus won't achieve the eye [2].

Regarding Herpes Zoster infection, according to Western Medicine, people over the age of 50 are particularly at risk, as the virus sustain itself on a weakened immune system to become active or re-active. Other factors are also accountable, such as HIV or any other diseases that affect the immune system, severe exhaustion, stress or anxiety, poor nutrition, chemotherapy or radiation therapy and chronic use of drugs such as corticosteroids or cyclosporine [5].

The treatment in Western Medicine is done with antiviral therapy. The treatment needs to be started as early as possible to be effective. Randomized trials have determined the clinical benefits of three antiviral medications: acyclovir, valacyclovir, and famciclovir [1- 6].
However, some patients, as those described in the case report, can present resistance to the treatment with antiviral drugs. Although this resistance is not common, affecting a small percentage of cases, it is usually related to mutations of one or two viral enzymes involved in the mechanism of action of the therapy with antiviral drugs [7].

In the two case studies presented in this article, the patients found an effective treatment in Traditional Chinese Medicine, without the use of antiviral drugs. According to the theories of Traditional Chinese Medicine, Herpes virus infections are linked to Heat retention in the Liver and Gallbladder's meridians [8].

Purpose

The purpose of this study, is, at first, to demonstrate the possibility of treatment of Herpes (both type 1, 2 and Zoster) without the use of antiviral drugs.

Second, to discuss and demonstrate the effectiveness of ancient medical tools, such as auricular acupuncture, apex ear bloodletting, Chinese dietary counselling, chakras measurement and high-diluted medications use in the treatment of this specific pathology.

Finally, to propose the treatment of Herpes and other pathologies reaching their root-level, considering the existence of energy imbalances, and reaffirming their importance in the treatment of the most varied pathologies.

\section{Methods}

This article was originated from the revision of the available literature on the treatment of Herpes infections in ancient medical traditions, as well as a summarized review of the treatment and diagnosis of the pathology in Western Medicine. All articles in the research are present in the Pubmed.

This literature review was done after the observation and study of two case reports that will be here exposed, in order to better explain the effectiveness of the treatment with ancient medical tools in the cases described.

\section{Case Study}

Patient C. M. was a two-year-old girl who presented a diagnosis of recurrent periocular Zoster Herpes, very close to her right eye. The patient's main symptom was excruciating painful red rashes on her face. Bullied on school, the pediatrician advised the mother 
to get the child a psychological treatment, worrying that the symptoms could be emotional. The family's main concern due to the symptom's recurrence was the possibility of the right eye's blindness.

Four years later, the now six-year-old patient started treatment with an Infectious Disease specialized doctor with a TCM background. The child's mother reported that the patient would experience around six crises of herpes within a year. I prescribed two sessions of auricular acupuncture associated with apex ear bloodletting per week, during times of crises, and once per week after the crises were resolved. The treatment was associated with Chinese dietary counselling.

The Chinese dietary orientations were to avoid fried foods, chocolate, eggs, coconut, honey, dairy products, especially when the cheese is melted, cold water, raw food and sweets. It was also recommend for the patient to avoid the consumption of pepper, cinnamon, ginger and cloves.

According to the patient's mother, the Traditional Chinese Medicine treatment was the "best things that ever happened in her daughter's life". The patient had extreme improvement in her daily life and the treatment diminished the crisis.

\section{Case Results}

The acupuncture treatment not only helped to prevent herpes attacks in the periocular region but also brought greater emotional serenity to the child who had problems with her parents' divorce, feeling less anxiety, and overall aiding in her physical and emotional development. The child did not have any crisis in the last 10 years.

\section{Case Report}

The patient S.E.M.M, a 68-year-old woman, with symptoms of recurrent genital herpes virus type 2 infection.

For four years the patient was constantly suffering with the emergence of genital herpes. The pathology caused great discomfort to the patient, together with pain, itching and redness.

After different allopathic treatments, with not impressive results, the patient decided to start treatment with acupuncture associated with apex ear bloodletting and Chinese Dietary Counselling and the results were completely outstanding for her.
The diet proposed was the same recommended in case 1 , but the major problem of the diet of this patient was the consumption of beer constantly on weekends. This factor is a major influence to the patient's condition.

\section{Case Results}

In a three-week period with the treatment instituted, the disease disappeared completely and did not relapse, until right now, a year and half later.

The patient reported to feel "free of the pain and discomfort caused by herpes, and completely thankful to the method used".

\section{Discussion}

Analyzing the two case studies, the author observed that the patients were treated holistically. In the first case report, it was possible to perceive improvement in different aspects of the subject's life: emotionally, in her development and growth as a child and physically, with the disappearance of the herpes symptoms and the strengthening of the patient's immune system, preventing other common infections during the period of acupuncture treatment.

In the second case, the patient had fast reduction of the genital herpes lesions through the energy-based treatment. It is possible that the constant use of high-concentrated, according to ArndtSchultz law better explained above, as well as the consumption of alcoholic beverages was inducing the formation of Heat in the Liver and Gallbladder meridians, leading to the genital herpes symptoms.

To understand better the effectiveness of the treatment described in the two case reports, the different methods used by the patients have to be understood.

This article analyzes three different spheres of their treatment. First, what was the treatment done with antiviral drugs, and the reason why this treatment was not effective in a long-term for both patients. Second, what is the theoretical base of the tools used in their treatment (auricular acupuncture, Chinese dietary counselling and apex ear bloodletting), and what is known in literature regarding these tools for treating this type of pathology.

Third and last, to comprehend how an energy-based treatment can have a positive effect in the quality of life of patients, as well as preventing new herpes outbreaks. 
Western Medicine Treatment: Oral acyclovir and virus resistance

It is well-established in literature that the treatment for herpes infections can be done with antiviral drugs, being successful in the majority of the cases.

For the three types of herpes (Herpes simplex type 1 and 2 and herpes Zoster), the treatment is usually done with the same drugs, acyclovir, valacyclovir and famciclovir, as many literature studies demonstrated, placing the treatment with this drug as the standard treatment [1-6, 8-10].

In the two case studies, the treatment with these drugs were not effective. The patient in case 1 maintained the treatment with oral acyclovir for years straight, until developing an allergy to the medication. With the use of the recipe prescribed, the patient still had around six herpes crises per year. In the second case report, the lesions would still persist with the use of the medication, or would disappear, but come back when the cycle of drug use ended.

Statically, the efficiency of the acyclovir is around $75 \%$ to $80 \%$, in reduction of the blisters, as well as avoiding recurrence of the lesions during and after the treatment [11].

When the treatment is not functionable, Western Medicine associates the fact with resistance of the virus. According to a study of 2003, from Teresa H. Bacon, entitled Herpes Simplex Virus Resistance to Acyclovir and Penciclovir after Two Decades of Antiviral Therapy, the number of patients with resistance to the drug is stable in around $0.3 \%$. This number can increase to $4 \%$ or $7 \%$ in immune compromised patients [12].

This small percentage of resistance to the antiviral reflects in the long-term use of the same medication, as presented in case 1. In this case, the patient presented resistance to the medication after the long-term use, and not effective results in the diminution of the blisters of herpes. The patient had periocular herpes, really close to her right eye.

When the disease affects the eye, the patient is at risk for more severe complications. These issues may include glaucoma, cataracts, double vision and scarring of the eyelid or cornea. That is why it was so important to treat the first patient as fast as possible, so the virus won't achieve the eye.

\section{Emotions and Herpes}

Several studies point out the relationship between emotional distress and the appearance of herpes. The justification lays on the fact that emotional distress affects the immune system negatively.

The relationship between emotions and the immune system were studied by several scholars, as, for example, Fulvio D'Acquisto, in the study entitled Affective immunology: where emotions and the immune response converge, states that a system not in balance will influence the immunological response, making it dysfunctional. Also, in this study, D'Aquisto will describe how further studies showed a "consistent association" between conscientiousness and reduced inflammatory response [13].

In another study, entitled Psychological Stress and the Human Immune System: A Meta-Analytic Study of 30 Years of Inquiry, from Suzzane C. Segestron, pointed out how chronic stress were associated with suppression of cellular measures, in 300 empirical articles [14].

An older study, of 1997, from John Green, entitled Psychological factors in recurrent genital herpes, established the relationship between self-esteem and the treatment of recurrent genital herpes, stating the recurrence of the disease could influence the psychological state, and that the opposite was also truth [14].

In the cases studied, the first patient was facing severe emotional distress, linked to her herpes symptoms as she was suffering bullying on school. On the other hand, the patient was facing her parents' divorce, what could be linked to the eruption of the herpes crises.

With the treatment instituted with auricular acupuncture, apex ear bloodletting and Chinese dietary counselling, the first patient presented herself as calmer and with more control of her general emotional state, at the same time, her herpes crises diminished, and the immune system of the patient also appeared to be stronger. Before the treatment, the patient constantly had flus and throat pain that also disappeared with the treatment, as she was physiologically stronger, and therefore, her immune system was stronger as well.

\section{Traditional Chinese Medicine and the emotions}

Traditional Chinese Medicine, on the other hand, places the emotions in the same logical scheme of the organs, according to the Five Elements theory. 
According to Traditional Chinese Medicine, and as previously explained by the author in other studies [16-21], the body is composed by different energies (Yin, Yang, Qi, Blood and Heat retention). These energies will have major influence in the body's health, and the disequilibrium between them will be the underlying cause of the pathologies and diseases, in this case, herpes.

According to extended analysis of Traditional Chinese Medicine physiopathology, diagnosis and treatment for herpes, it was possible to find that the main cause of herpes in the energy level is Heat retention in the Liver and Gallbladder's meridians [6].

These concepts will be better explained in the following section, but they are both severely influenced by the emotional state as well.

According to the Five Elements Theory, there are five massive organs that represent the five elements in the body, and are of primordial importance to the body's health. These organs are: Heart, Liver, Spleen, Kidneys and Lungs. Each of these organs are linked to one specific emotion, and have a hollow organ associated to them, as demonstrated in table $1[18,22]$.

\begin{tabular}{|l|l|l|l|l|l|}
\hline & Water & Wood & Fire & Earth & Metal \\
\hline Emotion & Fear & Anger & $\begin{array}{l}\text { Excessi- } \\
\text { ve Joy }\end{array}$ & Worry & Sadness \\
\hline $\begin{array}{l}\text { Hollow } \\
\text { Organ }\end{array}$ & Bladder & $\begin{array}{l}\text { Gallbla- } \\
\text { dder }\end{array}$ & $\begin{array}{l}\text { Small } \\
\text { Intestine }\end{array}$ & Stomach & $\begin{array}{l}\text { Large } \\
\text { Intestine }\end{array}$ \\
\hline $\begin{array}{l}\text { External } \\
\text { Sensorial } \\
\text { Organ }\end{array}$ & Ears & Eyes & Tongue & Mouth & Nose \\
\hline
\end{tabular}

Table 1: Five Elements Theory and the Emotions.

Repressed emotions can cause stagnant energy of the Liver and thus generate Heat. When consolidated, this Heat will appear in the body as a physical pathology. In this case, herpes.

In the table in table 1, as the Wood represents the energy of the Liver and have the Gallbladder as a hollow organ, the emotion that influence the formation of Herpes virus is anger [23].

The use of high-concentrated drugs and the drop in vital energy

The Arndt-Schultz law is a significant part of this and other studies of the author, and in this case, can also be used to under- stand why the treatment with antiviral therapy was not effective in the two case reports studied. According to the law, high-concentrated substances can decrease vital energy.

The law of Arndt-Schultz has been systemically studied in the medical field, supporting the theory of homeopathy and supposing many hazards can come with the excessive use of high-concentrated drugs, especially in a long term-use, influencing the general vital energy state and therefore the general states of the subject being treated.

For example, the study of Calabrese EJ, entitled Historical blunders: how toxicology got the dose-response relationship half right, explain how these misrepresentations of the Arndt-Schultz law impacted a broad range of governmental risk assessment activities further consolidating the rejection of hormesis [24].

Another study of from Domenico Mastrangelo, entitled Hormesis, epitaxy, the structure of liquid water, and the science of homeopathy, uses the Arndt-Schultz law to demonstrate the benefits of homeopathy use for not harming the energy of patients.

In Traditional Chinese Medicine, as previously said, the energies are believed to be the root of the health of a subject, therefore, any medications or substances that harm the energy of the patients should thus be avoided.

Their constant use, according to the TCM ancient theories would injury the Liver energy, leading to Internal Heat. They can also decrease vital energy, thus, generating more internal Heat, and contributing to the maintenance of the symptoms.

In the cases studied, both patients presented improvement in their general state of health, and diminution of their herpes symptoms after they withdrawn the high-concentrated drugs they were using.

Though acyclovir is a classic drug for herpes simplex virus infection, resistant strains to the drug have been found in immune-compromised patients and drug toxicity reports are commonly found.

The treatment instituted was more effective than the therapy with antivirals (in these two cases), and the reason to explain it can be linked to the use of high-concentrated drugs, and its influence in the health of the patient. 
Traditional Chinese Medicine and Herpes: The treatment instituted

Traditional Chinese Medicine comprehends diseases and symptoms as reflexes of imbalances in the energy. According to the theories, the body is composed by different energies (Yin, Yang, Qi and Blood), represented Figure 1, and different aspects and behaviors can influence the body (emotions, diet and external factors). Any imbalance in these energies will generate other imbalances, such as internal Heat, responsible for herpes symptoms [26].

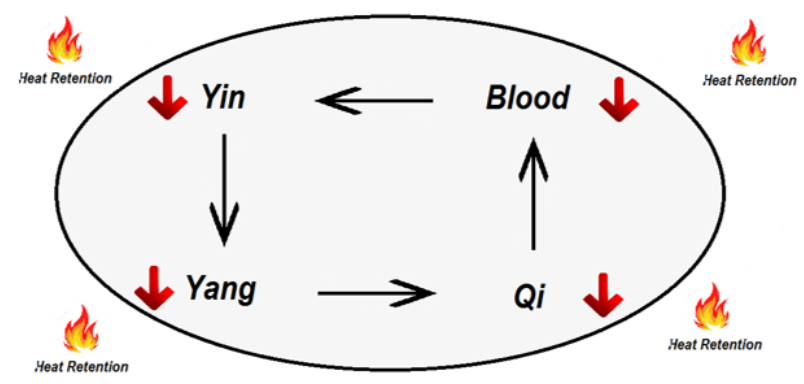

Figure 1: Yin, Yang, Qi and Blood.

The patients were also instructed to avoid common exposure to external cold and wind, such as taking baths with the doors and windows open, walking barefoot, sleeping with wet hair and not to sleep naked.

External factors have a significant influence in the health of the body. According to Traditional Chinese Medicine, external factors can penetrate the body, in an individual with energy deficiency, leading to imbalance in the energies, leading to formation of Heat, and worsening the herpes symptoms [26].

The treatment performed was individualized, according to the diagnosis of the energy imbalances, presented by the patients. It was focused in the energy imbalances of the patients, treating thus, the root of the problem and not fighting the pathogen itself. This concept of root and root-level was already explained in other articles of the author, through the metaphor of the tree [18-21].

In this metaphor, the author aims to explain the main differences between the treatment done in Western Medicine and the treatment done in Traditional Chinese Medicine for the most varied pathologies. The treatment in Western Medicine could be comprehended as a treatment in the leaf-level, where a symptom is treated according to a certain specialty, focused in the independent functions of the organs [18-21].

The scheme can be better comprehended in Figure 2.

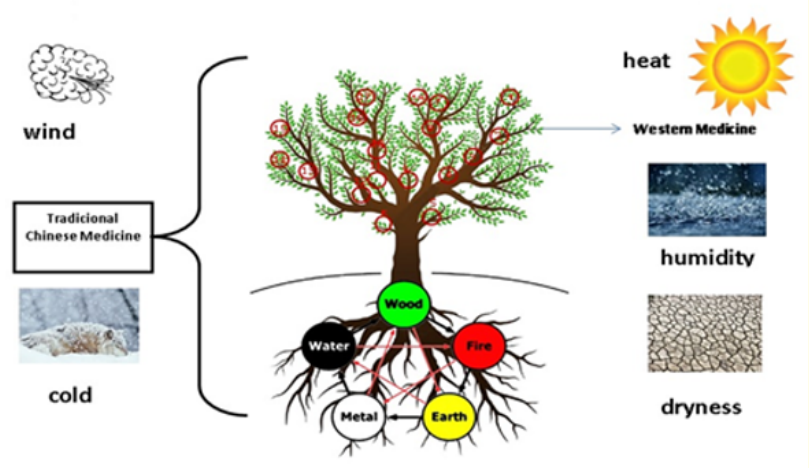

Figure 2: The metaphor of the tree.

The most important procedure to clean Heat is the apex ear bloodletting. [18-21] As in TCM different infectious diseases are associated with Internal Heat, apex ear bloodletting plays a significant role in the treatment of these kind of pathologies, including community and nosocomial infections.

The Liver is responsible for vision, according to the Five Elements Theory. This is why many times the herpes lesions will appear in the eye or near the eye, as their origin is the Liver, which is interlinked with the eye and with vision, as showed in table 1.

That is why besides changing the patient's diet, the patients received treatment for systemic energy imbalances, according to the imbalance diagnosis done in the first session, in order to rebalance the energy of Yin, Yang, Qi and Blood. The balance of these four major energies is responsible for health. When imbalanced, diseases may appear.

The effectiveness of Chinese dietary counselling has been well established in different articles and modern studies. Different scholars, such as Wu Q, in the study entitled Food therapy and medical diet therapy of Traditional Chinese Medicine, established the effectiveness of the Chinese dietary counselling in curing diseases [27]. 
Hippocrates, as demonstrated by the book of Elizabeth Craik, entitled Hippocratic Corpus, in which the author systematizes what was said by Hippocrates, states that there is a major importance on the diet in the treatment of a patient. According to Hippocrates, the power of nutriment is said to extern and influence all parts of the body. There is a whole chapter dedicated to food and nutrition [28].

Auricular acupuncture was also used, and was very important to the treatment of the both cases studied. The use of systemic and auricular acupuncture is being studied for the last couple of decades and is scientifically proved to have an influence in the immune system, and also to reduce inflammations [29].

Some examples of studies stating that would be The Anti-Inflammatory Actions of Auricular Point Acupressure for Chronic Low Back Pain, published by Wei-Chun Lee in 2015. In the study, it is stated: "Our findings strongly suggest that pain relief and improved physical function in patients with CLBP experienced through APA treatment may be modulated by the level of inflammatory cytokines" [29].

A study of 2012 from Abdi H, entitled Effects of auricular acupuncture on anthropometric, lipid profile, inflammatory, and immunologic markers: a randomized controlled trial study, perceived the same relation in his patients from a randomized study [30].

\section{Conclusion}

In this study, it was demonstrated that there is a possibility of treating recurrent herpes infection without the use of antiviral drugs. For this aim, it is necessary to see the patient as a whole, considering physical, emotional, dietary and external factors. Treating the energy imbalances of Yin, Yang, Qi and Blood, taking out Heat retention, accompanied by Chinese dietary counselling, it is possible to avoid the formation of Internal Heat, improving herpes recovery.

Other studies involving more patients should be done, but these two cases are examples to demonstrate the effectiveness of this method in recurrent herpes treatment.

As Hippocrates also said, "It is more important to know what sort of person has a disease than to know what sort of disease a person has".

\section{Bibliography}

1. Richard J WhitleyMD. "Herpes simplex virus infection". Seminars in Pediatric Infectious Diseases 13.1 (2002): 6-11.

2. Nelson Ls Lee, Ian Ct Tse. Herpes Simplex and Zoster. V. Major Opportunistic Infections.

3. Dahlia Saleh; Sandeep Sharma. Herpes Simplex Type 1. Treasure Island (FL): StatPearls Publishing (2019).

4. Sauerbrei. "Herpes Genitalis: Diagnosis, Treatment and Prevention". Geburtshilfe Frauenheilkd 76.12 (2016): 1310-1317.

5. Mary Jo Groves. Genital Herpes: A Review. American Family Physician.

6. Linda A. Waggoner-Fountain, Leigh B. Grossman. Herpes Simplex Virus.

7. Florence Morfin and Danielle Thouvenot. "Herpes simplex virus resistance to antiviral drugs". Journal of Clinical Virology 26.1 (2003): 29-37.

8. Huijuan Cao., et al. "Wet cupping therapy for treatment of herpes zoster: a systematic review of randomized controlled trials". Alternative Therapies In Health And Medicine 16.6 (2010): 48-54.

9. Dimitrios Kalogeropoulos., et al . "New Therapeutic Perceptions in a Patient with Complicated Herpes Simplex Virus 1 Keratitis: A Case Report and Review of the Literature". American Journal of Case Reports 18 (2017): 1382-1389.

10. Sonia Chaabane., et al . "Herpes simplex virus type 1 epidemiology in the Middle East and North Africa: systematic review, meta-analyses, and metaregressions". Scientific Reports (2019).

11. David W Kimberlin and Richard J Whitley. "Human Herpesviruses: Biology, Therapy, and Immunoprophylaxis. Chapter 64Antiviral therapy of HSV-1 and -2. Arvin A, CampadelliFiume G, Mocarski E, et al ., editors. Cambridge: Cambridge University Press (2007).

12. Teresa H Bacon., et al . "Herpes Simplex Virus Resistance to Acyclovir and Penciclovir after Two Decades of Antiviral Therapy". Clinical Microbiology Reviews16.1 (2003): 114-128.

13. Fulvio D’Acquisto. "Affective immunology: where emotions and the immune response converge". Dialogues in Clinical Neuroscience 19.1 (2017): 9-19. 
14. Suzzane C Segestron. "Psychological Stress and the Human Immune System: A Meta-Analytic Study of 30 Years of Inquiry". Psychological Bulletin 130.4 (2004): 601-630.

15. John Green and Agnes Kocsis. "Psychological factors in recurrent genital herpes”. Genitourinary medicine 73.4 (1997): 253258.

16. Huang Wei Ling. "Why do Patients Still Catch Hospital Infections Despite the Practice of Infection Prevention and Control Programs?". Acta Scientific Microbiology 1.4 (2018): 34-43.

17. Huang Wei Ling MD. "How Do You Treat Back Pain in Your Practice? Part 2". Medical Acupuncture 30.1 (2018).

18. Huang Wei Ling. "Why are Diabetic Patients Still Having Hyperglycemia despite Diet Regulation, Antiglycemic Medicati on and Insulin?". International Journal of Diabetes and Metabolic Disorders ISSN 2475-5451.

19. Huang Wei Ling. "Can Recurrent Furunculosis be Treated without the Use of Antibiotics?". Acta Scientific Microbiology 1.9 (2018): 04-12.

20. Huang Wei Ling. "Could Postsurgical Nosocomial Cellulitis be Treated without the Use of Antibiotics?". Acta Scientific Microbiology 1.9 (2018).

21. Huang W.L. "Can Hospital Osteomyelitis Be Treated Without the Use of Antibiotics?". Journal of Microbiology and Infectious Diseases 2.1(2018): 1-6.

22. Jagirdar PC. "The theory of five elements in acupuncture". The American Journal of Chinese Medicine 17.3-4 (1989): 135-138.

23. Christopher R Chase. "The Geometry of Emotions: Using Chakra Acupuncture and 5-Phase Theory to Describe Personality Archetypes for Clinical Use". Medical Acupuncture 30.4 (2018): 167-178.

24. Calabrese EJ. "Historical blunders: how toxicology got the dose-response relationship half right". Cellular and Molecular Biology (Noisy-le-grand) 51.7 (2005): 643-654.

25. Domenico Mastrangelo. "Hormesis, epitaxy, the structure of liquid water, and the science of homeopathy". (c) Medical Science Monitor 13.1 (2007): SR1-SR8.

26. OuYang B and Gu Z "Essentials of Traditional Chinese Medicine”. Shandong, Science and Technology Press (1996).
27. WU Q. "Food therapy and medical diet therapy of Traditional Chinese Medicine". Clinical Nutritional Experimental 18 (2018): 1-5.

28. Craik E. The Hippocratic Corpus: Content and Context. Routledge (2015).

29. Abdi H1., et al . "Effects of auricular acupuncture on anthropometric, lipid profile, inflammatory, and immunologic markers: a randomized controlled trial study". Journal of Alternative and Complementary Medicine 18.7 (2012): 668-677.

30. Wei-Chun Lee. "The Anti-Inflammatory Actions of Auricular Point Acupressure for Chronic Low Back Pain". Evidence-Based Complementary and Alternative Medicine (2015): 103570.

\section{Volume 3 Issue 8 August 2019 (C) All rights are reserved by Huang WL.}

